\title{
Review \\ Heat shock protein 60 reactive $T$ cells in juvenile idiopathic arthritis: what is new?
}

\author{
Yvonne Vercoulen ${ }^{1}$, Nienke H van Teijlingen ${ }^{1}$, Ismé M de Kleer ${ }^{1}$, Sylvia Kamphuis ${ }^{1}$, \\ Salvatore Albani ${ }^{2,3}$ and Berent J Prakken ${ }^{1,2}$
}

\author{
1'Department of Pediatric Immunology, Wilhelmina Children's hospital, UMCU, Lundlaan 6, 3584 EA, Utrecht, The Netherlands \\ ${ }^{2}$ Eureka Institute for Translational Medicine, Viale Teracati 50a, 96100, Siracusa, Italy \\ ${ }^{3}$ The University of Arizona College of Medicine, 1501 N. Campbell Avenue, PO BOX 245093, Tucson, AZ, USA
}

Corresponding author: Berent Prakken, bprakken@umcutrecht.nl

Published: 19 May 2009

This article is online at http://arthritis-research.com/content/11/3/231

(c) 2009 BioMed Central Ltd

\begin{abstract}
Juvenile idiopathic arthritis (JIA) is a disease characterized by chronic joint inflammation, caused by a deregulated immune response. In patients with JIA, heat shock proteins (HSPs) are highly expressed in the synovial lining tissues of inflamed joints. HSPs are endogenous proteins that are expressed upon cellular stress and are able to modulate immune responses. In this review, we concentrate on the role of HSPs, especially HSP60, in modulating immune responses in both experimental and human arthritis, with a focus on JIA. We will mainly discuss the tolerogenic immune responses induced by HSPs, which could have a beneficial effect in JIA. Overall, we will discuss the immune modulatory capacity of HSPs, and the underlying mechanisms of HSP60-mediated immune regulation in JIA, and how this can be translated into therapy.
\end{abstract}

\section{Introduction}

Juvenile idiopathic arthritis (JIA) is an autoimmune disease of unknown cause, characterized by a deregulated immune response in synovial lining tissues of the joints, leading to chronic arthritis in children. According to the latest classification provided by the International League of Associations for Rheumatology, seven categories of JIA can be distinguished, based on features present in the first 6 months of disease [1]. All forms of JIA are extensively described in a recent review by Ravelli and Martini [2]. Most of the literature we discuss in this review involves two of the most common forms: oligoarticular and polyarticular JIA. In the first 6 months, five or more joints are inflamed in polyarticular JIA, whereas only up to four joints are inflamed in oligoarticular JIA. Oligoarticular JIA can either be persistent or extended to five or more joints. The fact that persistent oligoarticular JIA is self-limiting, and in about half of all cases even self-remitting, suggests an endogenous regulation of the immune response, sometimes resulting in disease stabilization.

Heat shock proteins (HSPs) are endogenous proteins that are expressed upon cellular stress and are able to modulate immune responses. HSPs are highly present at sites of inflammation, like the inflamed joints of JIA patients [3] (Figure 1a).

Previously, we reviewed the options for specific immunotherapy in JIA using immune modulatory fragments of proteins, called peptides. This included some peptides derived from HSPs: HSP60 and the bacterial HSP dnaJ. These peptides were designed to be presented in major histocompatibility complex (MHC) II molecules, and are recognized by $\mathrm{T}$ cells. Thereby, these peptides enhance a specific immune response. The mechanisms and advantages of specific immunotherapy in JIA, compared to currently used immunosuppressive therapies, were extensively discussed [4].

In this review, we focus on the immune regulatory mechanisms of HSPS in arthritis, and, most importantly, JIA. Although we discuss other members of the family of HSPs, we concentrate on HSP60. First, we discuss the role of HSP60 in immune regulation. Second, we continue with immune regulation by HSP60 in experimental models of arthritis and rheumatoid arthritis (RA) and then the role of HSP60 in JIA. Last, we hypothesize on how the immune regulatory properties of HSP60 can be translated into therapy.

\section{Heat shock proteins}

HSPs are evolutionarily highly conserved proteins, either present constitutively, functioning as chaperones [5], or

$\mathrm{AA}=$ adjuvant arthritis; $\mathrm{DC}=$ dendritic cell; FOXP3 = forkhead box transcription factor $\mathrm{P} 3 ; \mathrm{HSP}=$ heat shock protein; IFN $=$ interferon; IL $=$ interleukin; JIA = juvenile idiopathic arthritis; $\mathrm{MHC}=$ major histocompatibility complex; RA = rheumatoid arthritis; TCR $=\mathrm{T}$ cell receptor; TLR $=$ Toll like receptor; $\mathrm{TNF}=$ tumour necrosis factor; $\operatorname{Tr} 1=\mathrm{T}$ regulatory $1 ; \operatorname{Treg}=$ regulatory $\mathrm{T}$ cell. 
(a)

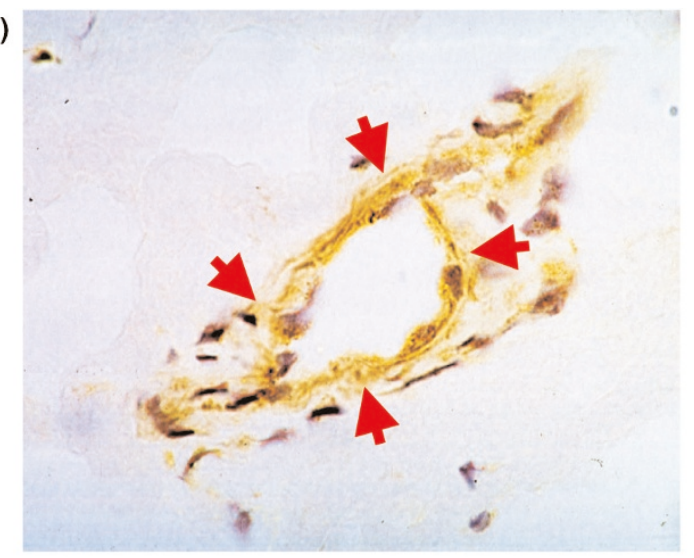

(b)

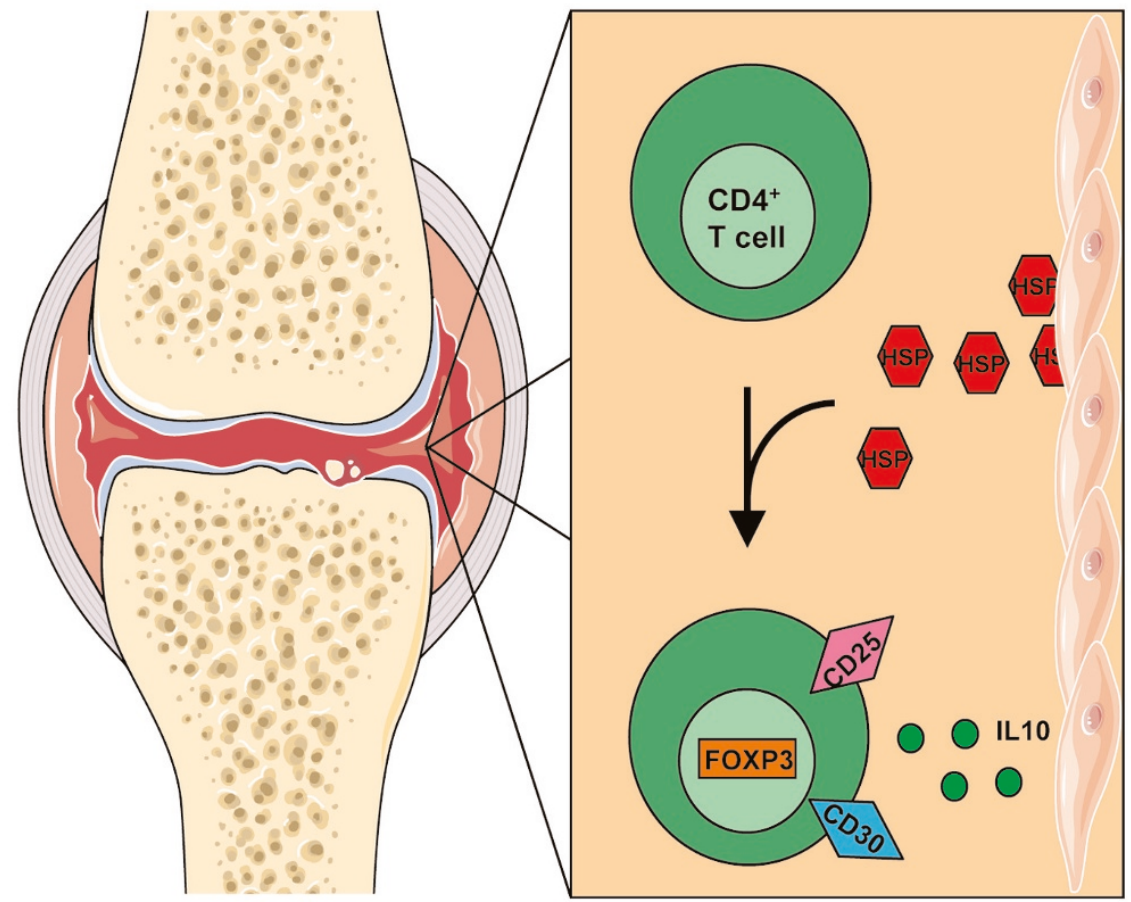

HSP60 specific T cells in the synovium of juvenile idiopathic arthritis (JIA) patients are IL-10-producing CD30+ regulatory T cell (Treg)-like cells. (a) HSP60 (stained brown and marked by red arrows) is highly expressed in synovial lining membranes in the inflamed joints of JIA patients. (b) HSP60 is released by the synovial tissues in the inflamed joint. In the synovial fluid, $C D 4^{+} \mathrm{T}$ cells are present. T cells that react to the selfHSP60 or HSP60 epitopes produce IL-10 $[13,68]$ and express CD30. Presence of these HSP60-reactive T cells correlates with a mild disease course [13]. Therefore, we hypothesize that these T cells could be CD25- and FOXP3-expressing naturally occurring Tregs [19], or IL-10producing T regulatory 1 cells. Altogether, HSP60 may induce Tregs in the joints of JIA patients and thereby regulate the inflammation of these JIA patients, as is seen in oligoarticular JIA. HSP, heat shock protein.

induced upon cell stress caused by, for instance, heat, oxidative stress, and hypoxia [6,7]. Several HSPs have been identified and, according to their size, organized into six families: HSP100, HSP90, HSP70, HSP60, HSP40, and HSP10.

In 1994 the 'danger model' was proposed by Polly Matzinger [8]. According to this model, the reaction of the immune response is not aimed towards 'self' or 'non-self', but towards 'danger'. Immune cells can sense danger when tissues that are stressed due to, for instance, infection release danger signals, such as HSPs. Therefore, an immune reaction is only elicited when necessary. As was discussed in a previous review, these danger signals also play a role in chronic inflammation [4]. As an example, in the inflamed joints of JIA patients, HSPs are released from the injured or dying cells in 
the synovial lining tissue and act as 'danger signals', alerting the immune system $[3,4]$. This does not mean that HSPs cause autoimmunity, but the increased expression and release of HSPs can have a major impact on the resulting immune response, as discussed below.

Indeed, different laboratories have documented variable immune responses towards HSPs in humans. In vitro experiments show that immune responses modulated by HSPs can be pro-inflammatory, for instance, by eliciting an interferon (IFN) $\gamma$ response by $\mathrm{T}$ cells, or tumour necrosis factor (TNF) $\alpha$ production by macrophages [9-11]. However, HSPs can also induce an anti-inflammatory response, for example, production of $\mathrm{LL}-10 \quad[12,13]$. HSPs are strong immune modulators and are able to influence the impact and direction of immune responses. In this review we will elaborate on the tolerogenic (anti-inflammatory) response, which, in different experimental settings, is attributed to an induction of IL-10 production. In the past decade, IL-10 producing $\mathrm{T}$ cells became more and more of interest. These cells belong to a population of $T$ cells - called regulatory T cells (Tregs) - that are key players in immune regulation $[14,15]$. HSPs may be able to influence this population of Tregs and thereby induce tolerogenic responses.

\section{Heat shock protein-mediated induction of tolerance: dependent on regulatory $\mathbf{T}$ cells? Regulatory T cells}

The involvement of HSPs in immune modulation, specifically in immune suppression, and $\mathrm{T}$ cell reactivity towards HSPs leading to suppression, raises the question of whether Tregs are involved in this process.

In the past decade, Tregs have been extensively investigated in several diseases. Their importance is clearly seen in patients suffering from IPEX (immune dysregulation polyendocrinopathy, enteropathy, $X$ chromosome-linked syndrome), who lack functional Tregs due to a deficiency in FOXP3 (Forkhead box transcription factor P3) [15,16]. As a consequence, these patients suffer from severe autoimmunity and allergies. The relevance of Tregs in regulation of autoimmunity has been confirmed by extensive research in several mouse models for diabetes and RA in which the transfer of Tregs to sick animals diminished disease [17,18]. Furthermore, the presence of Tregs correlated with a mild disease course in JIA, implicating a role for Tregs in controlling inflammation in JIA patients [19].

Subclasses of Tregs can be distinguished by their mechanism of suppression and phenotype. In general, suppression by Tregs is mediated by cell-cell contact and production of suppressive cytokines. Naturally occurring Tregs (nTregs), for instance, are present from birth and are distinguished by the expression of IL-2 receptor $\alpha$ (IL-2R $\alpha$; also called CD25) and FOXP3 [14]. T regulatory $1(\operatorname{Tr} 1)$ cells, however, are induced upon activation in peripheral tissues, and are characterized by production of IL-10 and IFN $\gamma$ [14]. It has been suggested that the population of activation-induced Tregs is the most important for immune regulation in humans $[20,21]$. In ex vivo and in vitro studies in humans it is difficult to differentiate between natural Tregs and induced Tregs. It is safe to assume that all HSP60-induced Tregs are such activationinduced Tregs; in the following, when using the term Tregs, we are thus referring to these cells.

\section{Regulatory T cell induction by HSP60}

There is indeed evidence that HSPs can contribute to an increase in Treg number and function. First of all, in animals with adjuvant arthritis (AA), treatment with HSP60 peptide and low dose anti-TNF $\alpha$ induced an increase in FOXP3expressing $\mathrm{T}$ cells, compared to treatment with high dose antiTNF $\alpha$ [22]. More in vitro data from patients with JIA suggest that Tregs are influenced by HSP60. In these patients the response of T cells to HSP60 [13], which correlated with a beneficial outcome of disease, was characterized by a high production of IL-10 and expression of the cell surface receptor CD30. CD30 is a member of the TNF receptor (TNFR) family and, in in vivo mouse experiments, was shown to be important for Treg function [23-25]. CD30 expression on $\mathrm{T}$ cells correlated with the production of T helper 2 cytokines and IL10 , but also with the expression of CD25, which is a hallmark of Tregs $[23,24]$ (Figure 1b). These CD30 expressing T cells could, therefore, represent a population of $\mathrm{T}$ cells that is able to regulate the immune response. A role for CD30-expressing $\mathrm{CD}^{+} \mathrm{T}$ cells in the regulation of human $\mathrm{T}$ helper 1 cell-driven diseases, like RA, has also been suggested [26,27]. We hypothesize that these $\mathrm{CD}^{+}{ }^{+} \mathrm{CD} 30^{+} \mathrm{T}$ cells could be important in the regulation of inflammation in JIA joints as well (Figure 1b). Future studies may elucidate whether, in inflamed joints, CD30-positive cells represent just activated cells, or cells with a possibly more regulatory phenotype.

Direct evidence showing an influence of HSPs on Tregs has been provided by in vitro experiments in which HSP6O and the HSP60 peptide p277 were able to enhance the regulatory function of the CD25-expressing Treg population from human peripheral blood, probably by increasing Treg migratory capacity $[28,29]$

\section{HSP-mediated immune modulation: a combination of innate and adaptive immunity}

Thus, as discussed above, HSP60 and HSP60-derived peptides seem to be able to enhance (Treg-mediated) immune suppression. The next important step would be to unravel the mechanism of Treg enhancement by HSP60. In this context, it is important to mention that HSP60 can trigger both adaptive and innate immune responses. HSP60 has been suggested several times to be a ligand for the Toll-like receptors (TLRs) 4 and 2 [11,30]. The TLRs belong to a family of highly conserved receptors that recognize molecular patterns of bacterial and viral origin, and form an important part of the innate defence. 
The production of some HSPs used for in vitro experiments takes place in bacteria, which means that there are always bacterial components contaminating them. For instance, microbial lipopolysaccharide, a ligand for TLR4, or microbial proteins such as peptidoglycan, a ligand for TLR2, are often detected in HSP preparations [31]. Therefore, to prevent interference caused by bacterial products or HSPs, it is best to focus on either highly purified HSPs, or on HSP peptides that are synthesized de novo.

HSP peptides may be expected to induce a specific T cellmediated response as their selection is based on $\mathrm{T}$ cell recognition. Interestingly, however, Cohen and co-workers [32] discovered that some HSP-derived peptides can also induce an innate immune response. They showed, as discussed before, that HSP60 peptide p277, which was originally identified as a peptide specifically recognized by $T$ cell clones [32], could enhance the population of Tregs. This was, however, mediated by TLR2 expressed on T cells [28]. Thus, apparently similar to the whole protein, HSP60-derived peptides are capable of activating both innate and adaptive immunity.

\section{Dendritic cells: linking innate and adaptive immunity}

The most direct link between the innate and adaptive immune system is formed by the professional antigen presenting cells, the dendritic cells (DCs). DCs express high levels of innate receptors, such as TLRs. Stimulation of these TLRs causes maturation of DCs. Conflicting data have been published regarding the maturity of DCs and their influence on Tregs; some data support that mature DCs are able to induce Tregs [33], while other publications suggest that specifically immature DCs can induce Tregs [34] (Figure 2a). The role of TLRs expressed on the DCs in the induction of Tregs has also been demonstrated to be controversial. On one hand, TLR stimulation on DCs can enhance IL-10 production by them, and thereby induction of Tregs (Figure 2a). On the other hand, stimulation of TLRs on DCs can lead to inhibition of Tregs and inflammation [35]. These data are mostly obtained from experiments in mice and in vitro experiments with human cells. Consistently, TLR agonists, tested in clinical trials as a treatment for tumours, do not always induce inflammation $[35,36]$. The direction of the immune response may depend on the intracellular signalling pathways that are induced [37]. Altogether, innate receptors can both directly and indirectly (via DC modulation) influence adaptive immunity and are able, in some cases, to contribute to the induction of tolerance through Tregs (Figure 2a). It seems likely that DCs, with their dual capacity to activate both through the innate and adaptive receptors, could play an essential role in the regulation of HSP60-mediated immunity. This has, however, not yet been studied in humans.

\section{Pathogen-associated molecular patterns enhance HSP-mediated tolerance}

In humans, memory $\mathrm{T}$ cells normally do not show a strong immune response to self-HSP60, but only to bacterial
HSP60, whereas naïve T cells may readily respond to both [38]. In addition, in cord blood, which comprises mainly naïve $T$ cells, responses to both human and bacterial HSP60 have been detected [39]. Low T cell responses to self-HSP60 and self-HSP60 peptides form an obstacle regarding their therapeutic applicability. For an optimal therapeutic effect, it seems logical to either enhance the self-HSP-mediated effect or to use the bacterial homologues of the human HSP6O peptides in order to induce a strong memory $\mathrm{T}$ cell response.

Thus, enhancement of this response can be achieved by combining the innate and adaptive immune system. The same principle is used in vaccinations: an adjuvant, like bacterial toxin, is used to elicit an immune response - in many cases via TLRs - that is sufficient to achieve immunological memory $[40,41]$. Furthermore, the innate immune response can be used to steer the antigen-specific immune response; TLRs can induce either a pro- or an anti- inflammatory reaction, as discussed above.

Altogether, as for vaccination, the combination of enhancing both adaptive and innate immunity may be attractive for immune therapy of arthritis. As discussed before, HSP60 may be able to do this on its own, as it can activate both the innate and adaptive immune systems (Figure 2b). Bacterial molecular patterns may enhance the effect of HSPs; for instance, a complex of lipopolysaccharide and HSP60, via TLR4 signalling in DCs, is able to enhance IL-12 production by DCs and IFN $\gamma$ production by $T$ cells [9]. In these experiments, the inflammatory response was enhanced via TLR4 on DCs. In contrast, there is evidence that bacterial patterns signalling via TLR2, TLR4 and TLR5 are able to enhance Treg function as well [42-44]. However, in this setting, the combination of bacterial patterns with HSPs or HSP peptides has not been investigated.

We hypothesize that the immune response can be modulated when T cell receptor (TCR) signalling is combined with TLR signalling: a synergy of innate and adaptive immunity. We propose that a combination of HSP peptides signalling through the TCR and possibly also TLRs together with pathogenic pattern signalling via TLRs could induce a stronger tolerogenic response (Figure 2c). Since HSPs can bind TLR2 and TLR4, their ligands, lipopolysaccharide and peptidoglycan, would be interesting targets. However, to achieve tolerance, the pathogenic patterns should be carefully tested in combination with HSP peptides to ensure that the immune response is skewed towards tolerance, not inflammation.

\section{Heat shock proteins in arthritis}

Several HSP families have been studied in relation to arthritis, either in animal or in vitro JIA and RA models, and some were even tested in vivo in clinical trials for RA. Although RA and JIA differ in, for instance, disease presentation and age of onset - RA onset occurs in adulthood, whereas JIA begins 
(a)

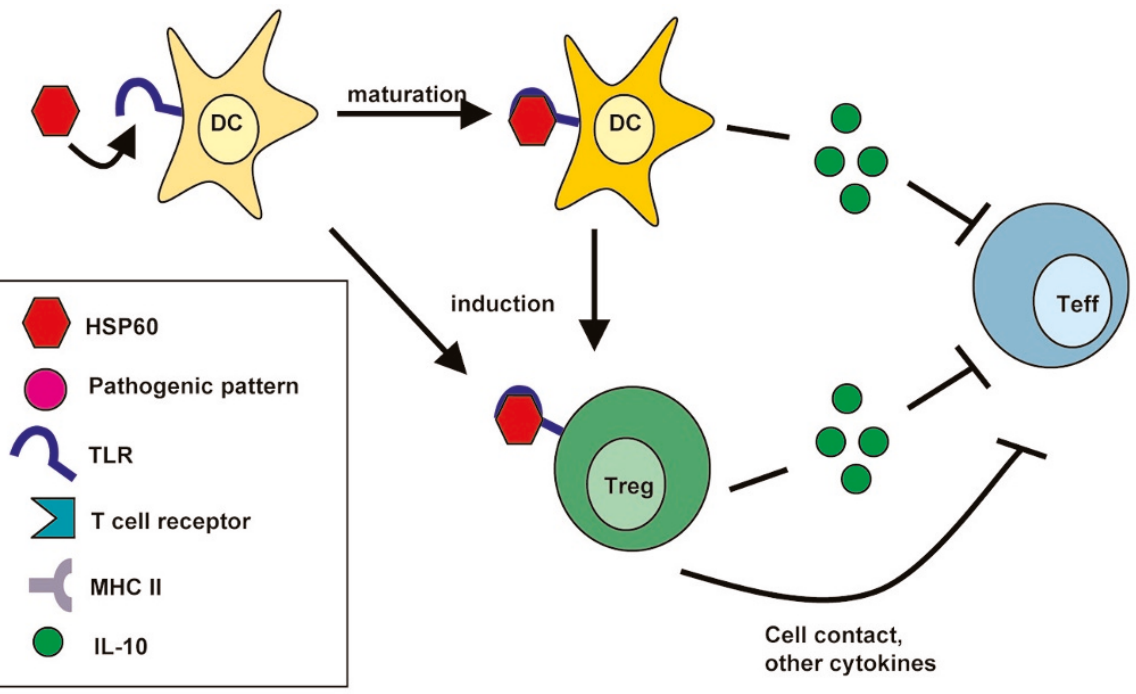

(b)

(c)
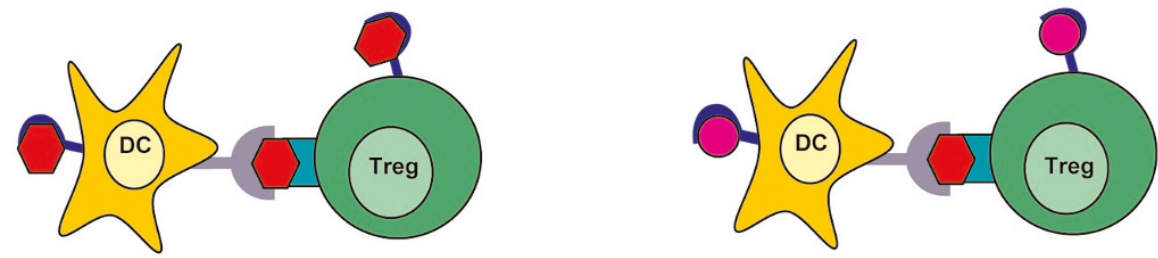

HSP60 uses both innate and adaptive immune pathways to enhance regulatory T cell (Treg) function. (a) HSP60 and HSP60-derived epitopes are able to bind to Toll-like receptors (TLRs). By binding to TLRs present on dendritic cells (DCs), HSP60 can induce maturation of DCs or cytokine production by them $[9,11]$, which could possibly cause induction of Tregs. Both DCs and Tregs are able to produce IL-10, which inhibits activation of effector T cells. To suppress the ongoing immune response, HSP60- induced Tregs could inhibit activation of effector T cells (Teffs) by cell-cell contact and/or production of IL-10, or other cytokines, such as transforming growth factor $\beta$ [14]. (b) Pan-HLA-DR (pan-DR) binding HSP60 epitopes are either presented to the Tregs in a major histocompatibility complex (MHC) II molecule by DCs, which could stimulate Tregs, or they are able to bind to TLRs on the Tregs [28] and thereby enhance Treg function. We hypothesize that HSP60 and HSP60-derived epitopes can enhance or induce Tregs by signalling through both the T cell receptor (TCR) and TLRs at the same time. HSP60 epitopes may bind directly to TLRs on the T cells, or to TLRs on the DCs, which could indirectly enhance the immune response. (c) We hypothesize that a combination of a TCR signal by a HSP60 pan-DR binding epitope and a TLR signal by a pathogenic pattern could enhance the HSP60-induced tolerogenic response, causing a stronger and longer lasting immune regulatory effect.

before 16 years of age - both are autoimmune diseases caused by a deregulated immune system $[2,45,46]$. Therefore, experimental findings in RA can also be useful for developing potential treatments for JIA.

The tolerogenic effect of HSPs on immune responses was discovered in experiments studying reactivity of $T$ cells towards bacterial and self-HSP in relation to arthritis. Several members of the HSP family are immunogenic in both experimental and human arthritis. Moreover, modulation of the HSP-specific response can restore immune tolerance in experimental arthritis; immunization with HSP10, HSP60, HSP70 or HSP90 can suppress experimental arthritis in a rat model of AA [47-50]. In humans, immune reactivity towards several HSPs is found in both peripheral blood and synovial fluid from patients with RA [51] and JIA [52,53]. In JIA, by far the most data available are on immune reactivity to human HSP60, which is discussed in depth below. Reactivity to other HSPs is described as well; for example, human homologues of peptides derived from the bacterial HSP dnaJ can induce tolerogenic IL-10 responses in synovial fluid cells of JIA patients [54].

In the context of RA, BiP, a member of the human HSP70 family, has drawn considerable attention. BiP is expressed in the synovium of RA patients, and is recognized by $T$ cells from RA patients [55]. These BiP-reactive $T$ cells showed a low proliferative response and IFN $\gamma$ production, but produced 
significant amounts of T helper 2 cytokines, such as IL-5 and IL-10. Furthermore, an anti-inflammatory gene profile was detected in monocytes after stimulation with BiP. These data suggest an immune modulatory role for BiP in RA. In a collagen-induced arthritis mouse model, similar results were found and, importantly, BiP was able to both prevent and treat arthritis [56]. Currently, the first clinical trials with $\mathrm{BiP}$ in $\mathrm{RA}$ are being planned.

The first HSP that was tested in the clinic as a treatment for $\mathrm{RA}$ is dnaJP1, a peptide derived from dnaJ. In a phase I clinical trial in patients with early RA, mucosal administration of dnaJP1 led to a shift in reactivity towards this peptide from predominant pro-inflammatory to a more tolerogenic immune response [52]. A recent placebo controlled phase II/III clinical trial reported clinical improvement in RA patients after oral treatment with dnaJP1 (EC Koffeman and S Albani, submitted for publication). In this clinical trial, HSP peptides were administered orally. Mucosal tissues are highly tolerant tissues, as they need to be able to accept exogenous antigens such as gut microbes and food. Therefore, encounters of the mucosal immune system with antigens often leads to 'oral tolerance' $[57,58]$. Oral tolerance can be achieved by induction of Tregs; for instance, mucosal administration of antigen can lead to the induction of IL-10-producing Tr1 cells, which eventually leads to tolerance towards the antigen [59]. This suggests that mucosal administration of antigens like HSPs could be beneficial for the induction of a tolerogenic response in arthritis. However, in both animal models and a clinical trial in type 1 diabetes other ways of HSP administration induced tolerance as well (reviewed by van Eden and colleagues [60]).

So far, one HSP60 epitope has been tested in a human autoimmune disease, diabetes. In a phase $\mathrm{lb} / \mathrm{ll}$ clinical trial, patients suffering from recent-onset type 1 diabetes were treated by subcutaneous injections with epitope p277, the same epitope that enhances Treg function in vitro [61]. Interestingly, these patients showed mainly IL-10 production in response to the HSP60 peptide, and clinical improvement was correlated with high IL-10 production before the start of therapy [62]. Although this is not the subject of this review, it underscores the potential of HSP60 peptides for treatment. It is crucial, however, to better understand the mechanism of HSP60-induced immune regulation before initiating clinical trials in arthritis.

Thus, altogether, HSPs are immune modulators in both experimental and human arthritis. Their common qualities are their increased expression in inflamed tissue, such as synovial tissue, and their ability to induce a tolerogenic response, which may have therapeutic applicability. From here on, this review concentrates on the most extensively studied HSP in arthritis, HSP60. First, we discuss results obtained from animal models, and then continue with the potential immune modulatory role of HSP60 in JIA.

\section{HSP60 in arthritis}

\section{HSP60 in experimental arthritis}

The studies on HSP60 in human arthritis originate from findings in the rat model of $A A$ in the late 1980s [63]. $A A$, an arthritis model with a close immunological and histopathological resemblance to RA and JIA, is induced by a single injection with heat-killed Mycobacterium tuberculosis in adjuvant.

First, it was found that $T$ cell clone $A 2 b$, the arthritogenic $T$ cell clone in AA, was reactive to an epitope derived from mycobacterial HSP65. Immunization with whole (myco-) bacterial HSP65, however, not only failed to induce arthritis, but also protected against the induction of $A A$ [63].

Next, Anderton and colleagues [64] demonstrated that T cells that were reactive towards bacterial HSP65 were responsible for the observed protection against the development of arthritis. Remarkably, protection from arthritis only occurred when peptides of bacterial HSP65 with a high degree of homology to self-HSP60 were used for immunization. This led to the induction of cross-reactive $\mathrm{T}$ cells recognizing both bacterial and human HSP [64].

This was, at the time, a stunning finding: it showed that, even in an autoimmune model of arthritis, $T$ cell reactivity to self-proteins did not necessarily lead to autoimmune inflammation. Moreover, T cell reactivity to self-HSP60 could lead to a tolerogenic response, contributing to protection from arthritis [64], as well as interference in ongoing arthritis [12]. The next obvious question is how this immune reactivity in experimental arthritis may relate to arthritis in humans.

\section{HSP60 in JIA}

It has turned out that the results obtained in AA are remarkably complementary to observations in the human autoimmune disease JIA. In inflamed joints from JIA patients, high levels of self-HSP60 are expressed in the synovial membranes [3] (Figure 1a). Furthermore, in these patients selfHSP60 is recognized by $T$ cells from the peripheral blood. Interestingly, when $T$ cell reactivity in synovial fluid from JIA patients was analyzed, those patients suffering from the mild and self-limiting oligoarticular form of JIA had an especially clear T cell response towards self-HSP60, while polyarticular patients did not [65]. This finding suggests a correlation between disease severity and HSP60 reactivity of T cells in these patients, in the sense that reactivity to self-HSP60 - like in the model of AA - may be beneficial. This has been further underscored in a prospective follow up study in new patients with JIA that demonstrated that T cell reactivity to self-HSP60 at the onset of disease was associated with a benign disease course [66]. Furthermore, self-HSP60 reactive T cells from synovial fluid express CD30 and produce IL-10 [13]. As discussed before, we suggest that these $T$ cells are Tregs (Figure 1b). 


\section{HSP60 epitopes: specific immune modulators in JIA}

Most of the discussed research has been performed using whole HSPs. As HSPs are immune-modulators, it is important to achieve the wished tolerogenic effect by selecting the appropriate stimulus. This may depend on which part of the protein is recognized by $T$ cells and elicits the immune response. Therefore, working with selected peptides derived from the whole protein increases specificity, and it probably reduces the chance of unwanted side effects. In experimental models of arthritis, T cell peptides from HSP60 are capable of having an effect with an efficacy that is similar to the whole protein. The problem, however, lies in the identification of such peptides in humans.

Humans, unlike the inbred animals used in experimental models, have a very heterogenic HLA background. Different HLA alleles have different binding affinities for peptides (epitopes) that can fit in their HLA peptide binding site. As a consequence, it is very difficult to determine which epitopes of HSP60 can bind HLA from an individual patient. Thus, predicting which epitopes are immunogenic in a heterogenic disease such as JIA is extremely complicated. This problem was overcome when, in collaboration with Alex Sette from the La Jolla Institute of Allergy and Immunology, a PanDR binding motif predicting computer algorithm that predicts binding to all HLA-DR genotypes was used [67]. We succeeded in selecting a set of HSP60-derived epitopes, both of human and bacterial origin, that were immunogenic in JIA, irrespective of the HLA background of the individual patients [68].

In further studies, it has been shown that human HSP60derived T cell epitopes, which were selected for their multiple MHC-genotype binding capacity, could both induce inflammatory cytokines and also regulatory cytokines like IL-10, depending on the specific epitope. Similar results have been demonstrated for some selected homologous bacterial HSP peptides. The induction of a tolerogenic response was detected in patients with several MHC-genotypes, which means that these peptides are able to induce beneficial responses in a large population of patients, independent of HLA background [68].

In the model of AA, the response to HSP60 has a dampening effect on chronic inflammation. As was shown in vitro for the HSP60 epitopes, this could also be the case for the response to HSP60 and HSP60 epitopes in JIA patients. Thus, HSP60 may contribute to the dampening of inflammation in the joints of JIA patients with a remitting course of disease. The identification of the HSP60 epitopes with a broad recognition in patients with JIA is a major step forward in the therapeutic application of HSP60 peptides in JIA.

\section{Tregs in JIA joints: unable to suppress inflammation?}

Many inflammatory cells are present in the joints of JIA patients. Due to inflammation, a high amount of self-HSP6O is released and, in addition, many FOXP3- and CD30-expressing $\mathrm{T}$ cells are present in the synovial fluid ( $\mathrm{Y}$ Vercoulen, manuscript in preparation; Figure 1b) Somehow, the presence of these $T$ cells is not sufficient to completely diminish inflammation. The 'cytokine signature' in synovial fluid from oligoarticular and polyarticular JIA patients confirmed these observations: IL-10 levels were higher in synovial fluid compared to plasma from JIA patients. However, even higher levels of pro-inflammatory cytokines were present in synovial fluid - for instance, IFN $\gamma$ and IL-6 [69].

The deficient immune regulation in the joints of JIA patients could be attributed to the Tregs themselves: the amount of Tregs may be insufficient, or the supposed Tregs may actually be activated effector $T$ cells that express FOXP3 transiently [70]. However, in in vitro assays the FOXP3+ Treg, isolated from the synovial fluid of JIA patients, can suppress activated $T$ cells ( $Y$ Vercoulen, manuscript in preparation). Furthermore, the frequency of FOXP3- and CD30-expressing $T$ cells is higher than in peripheral blood, whereas the suppressive capacity in vitro is comparable ( $Y$ Vercoulen, manuscript in preparation). Therefore, it is more likely that the local inflammatory environment in the joint influences Treg functionality, or makes activated $\mathrm{T}$ cells insusceptible to Treg-mediated suppression. Obviously, this local highly inflammatory state needs to be overcome before HSP60-mediated therapy, which may target the Treg population, can be applied,.

\section{Conclusion}

In this review we give an overview of the relevance of HSP60, and a few other HSPs, in JIA and immune modulation. We discuss that HSPs in general can induce a tolerogenic immune response, not only in vitro in human blood and synovial fluid cells from inflamed joints and in vivo in experimental models, but also in clinical trials in RA and type 1 diabetes. This tolerogenic response is marked by the induction of IL-10, an immune suppressive cytokine. Previous attempts at treatment with recombinant $\mathrm{IL}-10$ in clinical trials have not led to improvements in RA patients [71]. It has been suggested that IL-10, an immune suppressor, lacks efficacy [72]. We suggest that administration of a single cytokine is not representative for skewing of an immune response in which multiple cytokines are involved. HSPs probably not only increase IL-10 production by T cells, but also promote interactions of these cells with other immune cells, eventually leading to a tolerogenic response. Evidence for these suggestions has been provided in a recent publication, in which transgenic $\mathrm{IL}-10$-producing $\mathrm{T}$ cells, specific for auto-antigen, were transferred into mice suffering from proteoglycan-induced arthritis [73]. Although this is a very artificial setting, these $\mathrm{T}$ cells reduced arthritis, which was achieved by enhancing the endogenous tolerogenic response. Another advantage of inducing antigen-specific, IL-10producing $T$ cells is that they continue to produce IL-10 as long as they are activated by their specific antigen [74]; when inflammation decreases, less tissue proteins, such as HSPs, are released, and the regulatory response decreases as well. 
HSP60 is able to modulate a suppressive subset of cells, the Tregs [28]. The tolerogenic IL-10 responses that have been found in several experimental settings indicate that HSP60 may be able to induce IL-10-producing Tr1 cells. In JIA patients, HSP60 induces IL-10-producing $\mathrm{CD}^{+} \mathrm{T}$ cells, which express CD30, and the presence of these cells correlates with a mild course of disease [13]. Therefore, we hypothesize that $\mathrm{CD} 4{ }^{+} \mathrm{CD} 30^{+} \mathrm{T}$ cells are able to regulate the immune response in JIA. The suggested capacity of HSP60 to induce Tregs emphasizes the applicability of HSP60 (or peptides derived from HSP60) for immune therapy in arthritis.

HSP60 peptides influence Tregs via the TCR [68], or via TLRs [28], which are present on both $T$ cells and antigenpresenting cells such as DCs. This opens an intriguing possibility for immune therapy; to use one single peptide that may trigger antigen-specific $\mathrm{T}$ cells and may enhance this response by inducing innate immunity through TLR triggering at the same time.

Preliminary data suggest that a combination of both TCR and TLR activation causes induction of Tregs (De Kleer et al., manuscript in preparation). We hypothesize that Treg induction could even be enhanced by further augmenting innate immunity through TLR triggering by, for instance, pathogen-associated molecular patterns.

In humans, peripheral induction of Tregs is thought to be of high importance in the regulation of inflammation $[20,21]$. However, in JIA joints, many CD4 ${ }^{+}$CD $30{ }^{+}$FOXP3 $^{+} \mathrm{T}$ cells are present, which are functional Tregs in a neutral environment in vitro ( $\mathrm{Y}$ Vercoulen, manuscript in preparation). It is likely that the chronic inflammatory environment in the joints either causes local dysfunction of Tregs or makes activated cells insusceptible to Treg suppression. Therefore, suppression of inflammation is necessary before Treg targeted therapy can be applied.

\section{Towards therapy}

Thus, to create a therapeutic window for HSPs, it may be important to first dampen chronic inflammation in the joints for instance, by using anti-TNF $\alpha$ therapy [22]. Second, the combination of HSPs and modulators of innate immunity, such as pathogenic patterns, should be investigated more thoroughly. The combination of these two immune modulators may induce a stronger and longer lasting effect on the immune system by HSPs. However, both pathogenic patterns and HSPs can induce either tolerance or inflammation. Therefore, it is important to carefully select HSP epitopes and pathogenic patterns to ensure that they enhance only the tolerogenic effect and do not tip the delicate immune balance towards inflammation.

\section{Competing interests}

The authors declare that they have no competing interests.

\section{Acknowledgements}

YV is financially supported by the Dutch Rheumatoid Arthritis Foundation (Nationaal Reumafonds). BP is supported by the Dutch Rheumatoid Arthritis Foundation (Nationaal Reumafonds) and an NWO Innovation Impulse grant (VIDI) from the NWO.

\section{References}

1. Petty RE, Southwood TR, Manners P, Baum J, Glass DN, Goldenberg J, He X, Maldonado-Cocco J, Orozco-Alcala J, Prieur AM, Suarez-Almazor ME, Woo P: International League of Associations for Rheumatology classification of juvenile idiopathic arthritis: second revision, Edmonton, 2001. J Rheumatol 2004, 31:390-392.

2. Ravelli A, Martini A: Juvenile idiopathic arthritis. Lancet 2007, 369:767-778.

3. Boog CJ, de Graeff-Meeder ER, Lucassen MA, van der Zee R, Voorhorst-Ogink MM, van Kooten PJ, Geuze HJ, van Eden W: Two monoclonal antibodies generated against human hsp60 show reactivity with synovial membranes of patients with juvenile chronic arthritis. J Exp Med 1992, 175:1805-1810.

4. Koffeman EC, Prakken B, Albani S: Recent developments in immunomodulatory peptides in juvenile rheumatic diseases: from trigger to dimmer? Curr Opin Rheumatol 2005, 17:600605.

5. Craig EA, Gambill BD, Nelson RJ: Heat shock proteins: molecular chaperones of protein biogenesis. Microbiol Rev 1993, 57: 402-414.

6. Ellis RJ: The molecular chaperone concept. Semin Cell Biol 1990, 1:1-9.

7. Lindquist S, Craig EA: The heat-shock proteins. Annu Rev Genet 1988, 22:631-677.

8. Matzinger P: Tolerance, danger, and the extended family. Annu Rev Immunol 1994, 12:991-1045.

9. Osterloh A, Kalinke U, Weiss S, Fleischer B, Breloer M: Synergistic and differential modulation of immune responses by Hsp60 and lipopolysaccharide. J Biol Chem 2007, 282:4669.

10. Breloer M, More SH, Osterloh A, Stelter F, Jack RS, Bonin Av A: Macrophages as main inducers of IFN-gamma in T cells following administration of human and mouse heat shock protein 60. Int Immunol 2002, 14:1247-1253.

11. Ohashi K, Burkart V, Flohe S, Kolb H: Cutting edge: heat shock protein 60 is a putative endogenous ligand of the toll-like receptor-4 complex. J Immunol 2000, 164:558-561.

12. Prakken BJ, Roord S, van Kooten PJ, Wagenaar JP, van Eden W, Albani S, Wauben MH: Inhibition of adjuvant-induced arthritis by interleukin-10-driven regulatory cells induced via nasal administration of a peptide analog of an arthritis-related heatshock protein $60 \mathrm{~T}$ cell epitope. Arthritis Rheum 2002, 46: 1937.

13. de Kleer IM, Kamphuis SM, Rijkers GT, Scholtens L, Gordon G, de Jager W, Hafner R, van de Zee R, van Eden W, Kuis W, Prakken BJ: The spontaneous remission of juvenile idiopathic arthritis is characterized by CD30+ T cells directed to human heat-shock protein 60 capable of producing the regulatory cytokine interleukin-10. Arthritis Rheum 2003, 48:2001.

14. Vignali DA, Collison LW, Workman CJ: How regulatory $\mathrm{T}$ cells work. Nat Rev Immunol 2008, 8:523-532.

15. Sakaguchi S, Yamaguchi T, Nomura T, Ono M: Regulatory T cells and immune tolerance. Cell 2008, 133:775-787.

16. Bennett CL, Christie J, Ramsdell F, Brunkow ME, Ferguson PJ, Whitesell L, Kelly TE, Saulsbury FT, Chance PF, Ochs HD: The immune dysregulation, polyendocrinopathy, enteropathy, $X-$ linked syndrome (IPEX) is caused by mutations of FOXP3. Nat Genet 2001, 27:20.

17. You S, Leforban B, Garcia C, Bach JF, Bluestone JA, Chatenoud $\mathrm{L}$ : Adaptive TGF-beta-dependent regulatory $\mathrm{T}$ cells control autoimmune diabetes and are a privileged target of anti-CD3 antibody treatment. Proc Natl Acad Sci USA 2007, 104:6335.

18. Rodriguez-Palmero $M$, Franch $A$, Castell $M$, Pelegri $C$, Perez-Cano FJ, Kleinschnitz C, Stoll G, Hunig T, Castellote C: Effective treatment of adjuvant arthritis with a stimulatory CD28-specific monoclonal antibody. J Rheumatol 2006, 33:110.

19. de Kleer IM, Wedderburn LR, Taams LS, Patel A, Varsani H, Klein M, de Jager W, Pugayung G, Giannoni F, Rijkers G, Albani S, Kuis W, Prakken B: CD4+CD25(bright) regulatory $T$ cells actively regulate inflammation in the joints of patients with the remit- 
ting form of juvenile idiopathic arthritis. J Immunol 2004, 172: 6435.

20. Vukmanovic-Stejic M, Zhang Y, Cook JE, Fletcher JM, McQuaid A Masters JE, Rustin MH, Taams LS, Beverley PC, Macallan DC, Akbar AN: Human CD4+ CD25hi Foxp3+ regulatory T cells are derived by rapid turnover of memory populations in vivo. $J$ Clin Invest 2006, 116:2423.

21. Horwitz DA, Zheng SG, Gray JD: Natural and TGF-beta-induced Foxp3(+)CD4(+) CD25(+) regulatory $T$ cells are not mirror images of each other. Trends Immunol 2008, 29:429-435.

22. Roord ST, Zonneveld-Huijssoon E, Le T, Yung GP, Koffeman E, Ronaghy A, Ghahramani N, Lanza P, Billetta R, Prakken BJ, Albani $\mathrm{S}$ : Modulation of $\mathrm{T}$ cell function by combination of epitope specific and low dose anticytokine therapy controls autoimmune arthritis. PLOS ONE 2006, 1:e87.

23. Zeiser R, Nguyen VH, Hou JZ, Beilhack A, Zambricki EA, Buess $\mathrm{M}$, Contag $\mathrm{CH}$, Negrin RS: Early CD30 signalling is critical for adoptively transferred $C D 4+C D 25+$ regulatory $T$ cells in prevention of acute graft versus host disease. Blood 2006, 109: 2225-2233.

24. Dai Z, Li Q, Wang Y, Gao G, Diggs LS, Tellides G, Lakkis FG: CD4+CD25+ regulatory $T$ cells suppress allograft rejection mediated by memory CD8+ T cells via a CD30-dependent mechanism. J Clin Invest 2004, 113:310.

25. Blazar BR, Levy RB, Mak TW, Panoskaltsis-Mortari A, Muta $\mathrm{H}$, Jones M, Roskos M, Serody JS, Yagita H, Podack ER, Taylor PA: CD30/CD30 ligand (CD153) interaction regulates CD4+ T cell-mediated graft-versus-host disease. J Immunol 2004, 173:2933.

26. Gerli R, Lunardi C, Vinante F, Bistoni O, Pizzolo G, Pitzalis C: Role of CD30+ T cells in rheumatoid arthritis: a counter-regulatory paradigm for Th1-driven diseases. Trends Immunol 2001, 22:72.

27. Pellegrini $P$, Berghella AM, Contasta I, Adorno D: CD30 antigen: not a physiological marker for TH2 cells but an important costimulator molecule in the regulation of the balance between TH1/TH2 response. Transp/ Immuno/ 2003, 12:49.

28. Zanin-Zhorov A, Cahalon L, Tal G, Margalit R, Lider O, Cohen IR: Heat shock protein 60 enhances CD4+ CD25+ regulatory $T$ cell function via innate TLR2 signalling. J Clin Invest 2006, 116:2022.

29. Zanin-Zhorov A, Nussbaum G, Franitza S, Cohen IR, Lider O: T cells respond to heat shock protein 60 via TLR2: activation of adhesion and inhibition of chemokine receptors. FASEB $J$ 2003, 17:1567.

30. Vabulas RM, Ahmad-Nejad P, da Costa C, Miethke T, Kirschning CJ, Hacker $\mathrm{H}$, Wagner $\mathrm{H}$ : Endocytosed HSP60s use toll-like receptor 2 (TLR2) and TLR4 to activate the toll/interleukin-1 receptor signalling pathway in innate immune cells. J Biol Chem 2001, 276:31332-31339.

31. Tsan MF, Gao B: Heat shock protein and innate immunity. Cell Mol Immunol 2004, 1:274-279.

32. Elias D, Reshef T, Birk OS, van der Zee R, Walker MD, Cohen IR: Vaccination against autoimmune mouse diabetes with a $T$ cell epitope of the human $65-\mathrm{kDa}$ heat shock protein. Proc Natl Acad Sci USA 1991, 88:3088-3091.

33. Banerjee DK, Dhodapkar MV, Matayeva E, Steinman RM, Dhodapkar KM: Expansion of FOXP3high regulatory T cells by human dendritic cells (DCs) in vitro and after injection of cytokinematured DCs in myeloma patients. Blood 2006, 108:2655.

34. Roncarolo MG, Levings MK, Traversari C: Differentiation of T regulatory cells by immature dendritic cells. J Exp Med 2001, 193:F5-9.

35. Conroy $\mathrm{H}$, Marshall NA, Mills $\mathrm{KH}$ : TLR ligand suppression or enhancement of Treg cells? A double-edged sword in immunity to tumours. Oncogene 2008, 27:168-180.

36. Schmidt C: Clinical setbacks for toll-like receptor 9 agonists in cancer. Nat Biotechnol 2007, 25:825-826.

37. Hacker $\mathrm{H}$, Redecke V, Blagoev B, Kratchmarova I, Hsu LC, Wang GG, Kamps MP, Raz E, Wagner H, Hacker G, Mann M, Karin M: Specificity in Toll-like receptor signalling through distinct effector functions of TRAF3 and TRAF6. Nature 2006, 439:204207.

38. Ramage JM, Young JL, Goodall JC, Gaston JS: T cell responses to heat-shock protein 60: differential responses by CD4+ $T$ cell subsets according to their expression of CD45 isotypes. $J$ Immunol 1999, 162:704-710.
39. Merbl Y, Zucker-Toledano M, Quintana FJ, Cohen IR: Newborn humans manifest autoantibodies to defined self molecules detected by antigen microarray informatics. J Clin Invest 2007, 117:712-718.

40. Gupta RK, Siber GR: Adjuvants for human vaccines - current status, problems and future prospects. Vaccine 1995, 13: 1263-1276.

41. van Duin $D$, Medzhitov $R$, Shaw AC: Triggering TLR signalling in vaccination. Trends Immuno/ 2006, 27:49-55.

42. Liu H, Komai-Koma M, Xu D, Liew FY: Toll-like receptor 2 signalling modulates the functions of CD4+ CD25+ regulatory $\mathrm{T}$ cells. Proc Natl Acad Sci USA 2006, 103:7048.

43. Caramalho I, Lopes-Carvalho T, Ostler D, Zelenay S, Haury M, Demengeot J: Regulatory $\mathrm{T}$ cells selectively express toll-like receptors and are activated by lipopolysaccharide. J Exp Med 2003, 197:403.

44. Crellin NK, Garcia RV, Hadisfar O, Allan SE, Steiner TS, Levings MK: Human CD4+ T cells express TLR5 and its ligand flagellin enhances the suppressive capacity and expression of FOXP3 in CD4+CD25+ T regulatory cells. J Immunol 2005, 175:8051. 8059.

45. Dewint $\mathrm{P}$, Hoffman IE, Rogge $\mathrm{S}$, Joos R, Union A, Dehoorne J, Delanghe J, Veys EM, De Keyser F, Elewaut D: Effect of age on prevalence of anticitrullinated protein/peptide antibodies in polyarticular juvenile idiopathic arthritis. Rheumatology 2006 45:204-208.

46. Wagner-Weiner L: Pediatric rheumatology for the adult rheumatologist. J Clin Rheumato/ 2008, 14:109-119.

47. Wendling U, Paul L, van der Zee R, Prakken B, Singh M, van Eden W: A conserved mycobacterial heat shock protein (hsp) 70 sequence prevents adjuvant arthritis upon nasal administration and induces IL-10-producing T cells that cross-react with the mammalian self-hsp70 homologue. J Immunol 2000, 164: 2711-2717.

48. Tanaka S, Kimura $Y$, Mitani A, Yamamoto G, Nishimura $H$, Spallek $\mathrm{R}$, Singh M, Noguchi T, Yoshikai $Y$ : Activation of T cells recognizing an epitope of heat-shock protein 70 can protect against rat adjuvant arthritis. J Immunol 1999, 163:5560-5565.

49. Quintana FJ, Carmi P, Mor F, Cohen IR: Inhibition of adjuvantinduced arthritis by DNA vaccination with the 70-kd or the 90kd human heat-shock protein: immune cross-regulation with the 60-kd heat-shock protein. Arthritis Rheum 2004, 50:37123720.

50. Ragno S, Winrow VR, Mascagni $P$, Lucietto $P$, Di Pierro F, Morris CJ, Blake DR: A synthetic 10-kD heat shock protein (hsp10) from Mycobacterium tuberculosis modulates adjuvant arthritis. Clin Exp Immunol 1996, 103:384-390.

51. Roelofs MF, Boelens WC, Joosten LA, Abdollahi-Roodsaz S, Geurts J, Wunderink LU, Schreurs BW, van den Berg WB, Radstake TR: Identification of small heat shock protein B8 (HSP22) as a novel TLR4 ligand and potential involvement in the pathogenesis of rheumatoid arthritis. J Immunol 2006, 176:7021-7027.

52. Albani S, Ravelli A, Massa M, De BF, Andree G, Roudier J, Martini A, Carson DA: Immune responses to the Escherichia coli dnaJ heat shock protein in juvenile rheumatoid arthritis and their correlation with disease activity. J Pediatr 1994, 124:561.

53. De Graeff-Meeder ER, van der Zee R, Rijkers GT, Schuurman HJ, Kuis W, Bijlsma JW, Zegers BJ, van Eden W: Recognition of human $60 \mathrm{kD}$ heat shock protein by mononuclear cells from patients with juvenile chronic arthritis. Lancet 1991, 337:13681372.

54. Massa M, Passalia M, Manzoni SM, Campanelli R, Ciardelli L, Yung GP, Kamphuis S, Pistorio A, Meli V, Sette A, Prakken B, Martini A, Albani S: Differential recognition of heat-shock protein dnaJ-derived epitopes by effector and Treg cells leads to modulation of inflammation in juvenile idiopathic arthritis. Arthritis Rheum 2007, 56:1648.

55. Blass S, Union A, Raymackers J, Schumann F, Ungethum U, Muller-Steinbach S, De Keyser F, Engel JM, Burmester GR: The stress protein BiP is overexpressed and is a major $B$ and $T$ cell target in rheumatoid arthritis. Arthritis Rheum 2001, 44: 761-771.

56. Panayi GS, Corrigall VM: BiP regulates autoimmune inflammation and tissue damage. Autoimmun Rev 2006, 5:140-142.

57. Weiner HL: Oral tolerance, an active immunologic process mediated by multiple mechanisms. J Clin Invest 2000, 106: 
935-937.

58. Mowat AM: Anatomical basis of tolerance and immunity to intestinal antigens. Nat Rev Immuno/ 2003, 3:331-341.

59. Battaglia M, Gianfrani C, Gregori S, Roncarolo MG: IL-10-producing $\mathrm{T}$ regulatory type 1 cells and oral tolerance. Ann $N$ Y Acad Sci 2004, 1029:142-153.

60. van Eden W, van der Zee R, Prakken B: Heat-shock proteins induce T-cell regulation of chronic inflammation. Nat Rev Immunol 2005, 5:318.

61. Huurman VA, Decochez K, Mathieu C, Cohen IR, Roep BO: Therapy with the hsp60 peptide DiaPep277 in C-peptide positive type 1 diabetes patients. Diabetes Metab Res Rev 2007, 23:269-275.

62. Huurman VA van der Meide PE, Duinkerken $G$, Willemen $S$, Cohen IR, Elias D, Roep BO: Immunological efficacy of heat shock protein 60 peptide DiaPep277 therapy in clinical type I diabetes. Clin Exp Immunol 2008, 152:488-497.

63. van Eden W, Thole JE, van der Zee R, Noordzij A, van Embden JD, Hensen EJ, Cohen IR: Cloning of the mycobacterial epitope recognized by $\mathrm{T}$ lymphocytes in adjuvant arthritis. Nature 1988, 331:171-173.

64. Anderton SM, van der Zee R, Prakken B, Noordzij A, van Eden W: Activation of $T$ cells recognizing self $60-k D$ heat shock protein can protect against experimental arthritis. J Exp Med 1995, 181:943-952.

65. de Graeff-Meeder ER, van EW, Rijkers GT, Prakken BJ, Kuis W, Voorhorst-Ogink MM, van der ZR, Schuurman HJ, Helders PJ, Zegers BJ: Juvenile chronic arthritis: T cell reactivity to human HSP60 in patients with a favorable course of arthritis. J Clin Invest 1995, 95:934.

66. Prakken $A B$, van Eden W, Rijkers GT, Kuis W, Toebes EA, de Graeff-Meeder ER, van der Zee R, Zegers BJ: Autoreactivity to human heat-shock protein 60 predicts disease remission in oligoarticular juvenile rheumatoid arthritis. Arthritis Rheum 1996, 39:1826-1832.

67. Sette A: Tools of the trade in vaccine design. Science 2000 , 290:2074-2075.

68. Kamphuis S, Kuis W, de JW, Teklenburg G, Massa M, Gordon G, Boerhof M, Rijkers GT, Uiterwaal CS, Otten HG, Sette A, Albani $\mathrm{S}$, Prakken BJ: Tolerogenic immune responses to novel T-cell epitopes from heat-shock protein 60 in juvenile idiopathic arthritis. Lancet 2005, 366:50.

69. de Jager W, Hoppenreijs EP, Wulffraat NM, Wedderburn LR, Kuis $W$, Prakken BJ: Blood and synovial fluid cytokine signatures in patients with juvenile idiopathic arthritis: a cross-sectional study. Ann Rheum Dis 2007, 66:589-598.

70. Wang J, loan-Facsinay A van dV, Huizinga TW, Toes RE: Transient expression of FOXP3 in human activated nonregulatory CD4(+) T cells. Eur J Immunol 2007, 37:129.

71. Smeets TJ, Kraan MC, Versendaal J, Breedveld FC, Tak PP: Analysis of serial synovial biopsies in patients with rheumatoid arthritis: description of a control group without clinical improvement after treatment with interleukin 10 or placebo. $J$ Rheumatol 1999, 26:2089-2093.

72. Asadullah K, Sterry W, Volk HD: Interleukin-10 therapy - review of a new approach. Pharmacol Rev 2003, 55:241-269.

73. Guichelaar T, ten Brink CB, van Kooten PJ, Berlo SE, Broeren CP, van Eden W, Broere F: Autoantigen-specific IL-10-transduced T cells suppress chronic arthritis by promoting the endogenous regulatory IL-10 response. J Immunol 2008, 180:13731381.

74. Walker LS, Chodos A, Eggena M, Dooms H, Abbas AK: Antigendependent proliferation of CD4+ CD25+ regulatory T cells in vivo. J Exp Med 2003, 198:249-258. 